\title{
Duality of Metric Entropy in Euclidean Space
}

\author{
S. Artstein *, V. D. Milman *, S. J. Szarek ${ }^{\dagger}$
}

\begin{abstract}
Let $K$ be a convex body in a euclidean space, $K^{\circ}$ its polar body and $D$ the euclidean unit ball. We prove that the covering numbers $N(K, t D)$ and $N\left(D, t K^{\circ}\right)$ are comparable in the appropriate sense, uniformly over symmetric convex bodies $K$, over $t>0$ and over the dimension of the space. In particular this verifies the duality conjecture for entropy numbers of linear operators, posed by Pietsch in 1972 , in the central case when either the domain or the range of the operator is a Hilbert space.
\end{abstract}

For two convex bodies $K$ and $T$ in $\mathbb{R}^{n}$, the covering number of $K$ by $T$, denoted $N(K, T)$, is defined as the minimal number of translates of $T$ needed to cover $K$

$$
N(K, T)=\min \left\{N: \exists x_{1} \ldots x_{N} \in \mathbb{R}^{n}, K \subset \bigcup_{i \leq N} x_{i}+T\right\} .
$$

We denote by $D$ the euclidean unit ball in $\mathbb{R}^{n}$. In this paper we announce and sketch the proof of the following duality result for covering numbers. [The details will appear elsewhere.]

Theorem 1 There exist two universal constants $\alpha$ and $\beta$ such that for any dimension $n$, any convex body $K \subset \mathbb{R}^{n}$ symmetric with respect to the origin and any $t>0$, one has

$$
N\left(D, \alpha t K^{\circ}\right)^{\frac{1}{\beta}} \leq N(K, t D) \leq N\left(D, \alpha^{-1} t K^{\circ}\right)^{\beta}
$$

where $K^{\circ}:=\left\{u \in \mathbb{R}^{n}: \sup _{x \in K}\langle x, u\rangle \leq 1\right\}$ is the polar body of $K$.

The best constant $\beta$ that our approach yields is $\beta=2+\varepsilon$ for any $\varepsilon>0$, with $\alpha=\alpha(\varepsilon)$ (i.e., with $\alpha$ depending on $\varepsilon$ and only on $\varepsilon$ ).

Theorem 1 resolves an old problem, going back to Pietsch ([P], p. 38) and referred to as the "duality conjecture for entropy numbers", in a special yet most important case. The problem can be stated in terms of covering numbers in the following way (below and in what follows we shall abbreviate "symmetric with respect to the origin" to just "symmetric").

\footnotetext{
${ }^{*}$ School of Mathematical Sciences, Tel Aviv University, Tel Aviv 69978, Israel ${ }^{\dagger}$ Equipe d'Analyse Fonctionnelle, B.C. 186, Université Paris VI, 4 Place Jussieu, F-75252 Paris, France and Department of Mathematics, Case Western Reserve University, Cleveland, OH 44106-7058, U.S.A. E-mail addresses: artst@post.tau.ac.il,milman@post.tau.ac.il, szarek@ccr.jussieu.fr
} 
Conjecture 2 Do there exist two numerical constants $a, b \geq 1$ such that for any dimension $n$, and for any two symmetric convex bodies $K$ and $T$ in $\mathbb{R}^{n}$ one has

$$
\log N\left(T^{\circ}, a K^{\circ}\right) \leq b \log N(K, T),
$$

where $A^{\circ}$ denotes the polar body of $A$ ?

Theorem 1 verifies this conjecture in the case where one of the two bodies is a euclidean ball or, more generally, by affine invariance of the problem, when one of the two bodies is an ellipsoid. [Note that the additional parameter $t$ present in the statement of Theorem 1 does not affect the generality.] In the special case where both bodies are ellipsoids it is well known and easy to check that there is equality in (2), with $a=b=1$.

The quantity $\log N(K, t T)$ has a clear information-theoretic interpretation: it is the complexity of $K$ (measured in bits if the logarithm is to the base 2) at the level of resolution $t$ with respect to the metric associated with $T$ (e.g., euclidean if $T=D$ ). Accordingly, (1) means that the complexity of $K$ in the euclidean sense is controlled by that of the euclidean ball with respect to $\|\cdot\|_{K^{\circ}}$ (the gauge of $K^{\circ}$, i.e., the norm whose unit ball is $K^{\circ}$ ), and vice versa, at every level of resolution. While it is clear that these two complexities should be related, the universality of the link that we establish is somewhat surprising. The covering numbers appear also in many other areas of mathematics, for example both quantities $N(K, t D)$ and $N\left(D, t K^{\circ}\right)$ enter the theory of Gaussian processes (see, e.g., the survey $[\mathrm{L}]$ and its references).

Conjecture 2 originated in operator theory, and so we restate it below in the language of entropy numbers of operators. For two Banach spaces $X$ and $Y$, with unit balls $B(X)$ and $B(Y)$ respectively, and for a linear operator $u: X \rightarrow Y$, the $k^{t h}$ entropy number of $u$ is defined by

$$
e_{k}(u):=\inf \left\{\varepsilon: N(u B(X), \varepsilon B(Y)) \leq 2^{k-1}\right\} .
$$

Considering the entropy numbers of the dual operator $u^{*}: Y^{*} \rightarrow X^{*}$ means covering $(B(Y))^{\circ}$ with (translates of) $\varepsilon(B(X))^{\circ}$. One sees easily that $e_{1}(u)=\|u\|_{o p}$ (the operator norm), and so $e_{1}(u)=e_{1}\left(u^{*}\right)$; similarly $e_{k}(u) \rightarrow 0($ as $k \rightarrow \infty)$ iff $e_{k}\left(u^{*}\right) \rightarrow$ 0 (iff $u$ is a compact operator). As the sequence $\left(e_{k}(\cdot)\right)$ quantifies the compactness of an operator, it is natural to ask to what extent $\left(e_{k}(u)\right)$ and $\left(e_{k}\left(u^{*}\right)\right)$ behave similarly. This is the context in which the original duality conjecture was formulated, and it read as follows.

Conjecture 2A (Duality Conjecture in the language of entropy numbers). - Do there exist numerical constants $a, b \geq 1$, such that for any two Banach spaces $X$ and $Y$, any linear operator $u: X \rightarrow Y$ and any natural number $k$, one has

$$
e_{b k}\left(u^{*}\right) \leq a e_{k}(u) ?
$$

Our Theorem 1 verifies thus the duality conjecture (when expressed in terms of entropy numbers) in the case when one of the two spaces, either the domain or the range of the operator $u$, is a Hilbert space. [The passage from finite- to infinite-dimensional setting is achieved by a straightforward approximation once dimension-free estimates 
are available.] Some special cases of the problem have been studied before, and some particular results were established, see [AMS] for references. The known results which we need for our proof are those from $[\mathrm{A}],[\mathrm{KM}],[\mathrm{MS}]$ and $[\mathrm{T}]$.

Our proof consists of three main steps. The first is a duality result involving the diameter of the set. In fact we have two alternative first steps, the first of which was shown in [AMS]: denoting $\psi(x)=C_{0}(\log x)^{3}$ for some appropriate numerical constant $C_{0}>0$, we have

Lemma 3 If $K \subset R D \subset \mathbb{R}^{n}$ is a convex symmetric body then

$$
\begin{aligned}
N\left(D, \psi(R) K^{\circ}\right) & \leq N(K, D)^{2} \\
N(K, D) & \leq N\left(D,(1 / \psi(R)) K^{\circ}\right)^{3} .
\end{aligned}
$$

The other alternative for the first step is the following fact, the proof of which is significantly easier than the proof of Lemma 3 . However, there is a price to pay for simplifying the argument: instead of $\beta=2+\varepsilon$ in the exponent in (1), we get some other, perhaps huge, universal constant.

Lemma 4 There exist universal constants $c_{3}$ and $C_{4}$ such that for any dimension $n$, and for any symmetric convex body $K \subset R D \subset \mathbb{R}^{n}$, we have

$$
N(K, D) \leq N\left(D, c_{3} K^{\circ}\right)^{C_{4} R^{2}} \text { and } \quad N\left(D, K^{\circ}\right) \leq N\left(K, c_{3} D\right)^{C_{4} R^{2}} .
$$

With Lemma 4 (or 3) at hand, we come to the second step which is an iteration procedure. At each step of the iteration we truncate the body at a certain radius, and use elementary geometric inequalities for covering numbers. (In the dual inequality at each step we add a small ball to the body). We thus bound a covering number by a product of such numbers, where for each factor in the product we have control on the diameter of the respective body.

Lemma 5 For any symmetric convex body $K \subset \mathbb{R}^{n}$ and any sequence $R_{0}<R_{1}<$ $\cdots<R_{s}$

$$
\begin{aligned}
N\left(D, R_{0} K^{\circ}\right) & \leq N\left(D, R_{s} K^{\circ}\right) \prod_{j=0}^{s-1} N\left(D, \frac{R_{j}}{2}\left(K \cap R_{j+1} D\right)^{\circ}\right) \\
N\left(K, R_{0} D\right) & \leq N\left(K, R_{s} D\right) \prod_{j=0}^{s-1} N\left(2 K \cap R_{j+1} D, R_{j} D\right) .
\end{aligned}
$$

We choose the sequence $R_{j}$ to be a geometric progression of the form $R_{j}=C_{1} C_{2}^{j}$, for big enough (but universal) constants $C_{1}$ and $C_{2}$. Lemma 4 and Lemma 5 imply now the following 
Corollary 6 With the above choice of the sequence $\left(R_{j}\right)$ we have, for every symmetric convex body $K$,

$$
\begin{aligned}
N\left(D, R_{0} K^{\circ}\right) & \leq N\left(D, R_{s} K^{\circ}\right) \prod_{j=0}^{s-1} N\left(K \cap R_{j+1} D, \frac{c_{3}}{2} R_{j} D\right)^{4 C_{4} C_{2}^{2}} \\
N\left(K, R_{0} D\right) & \leq N\left(K, R_{s} D\right) \prod_{j=0}^{s-1} N\left(D, c_{3} R_{j}\left(2 K \cap R_{j+1} D\right)^{\circ}\right)^{C_{4} C_{2}^{2}} .
\end{aligned}
$$

If we use Lemma 3 instead of Lemma 4 and an appropriate sequence $R_{j}$, we can prove a similar corollary with better constants in the exponent, 2 and 3 respectively, which with still more work - can be improved to $1+\varepsilon$ in both inequalities.

In the third and last step we collapse the long products of covering numbers appearing in (7) and (8) to products consisting of just several terms. The largest $R_{s}$ will be chosen to exceed the diameter of the set $K$, and so the terms $N\left(K, R_{s} D\right)$ and $N\left(D, R_{s} K^{\circ}\right)$ will both equal to 1 . We use the following two super-multiplicativity properties of covering numbers.

Lemma 7 Let $R>r>5 S>5 s$. Then

$$
\begin{aligned}
N(K \cap R D, r D) N(K \cap S D, s D) & \leq N\left(K \cap R D, \frac{s}{4} D\right) \\
N\left(D, r(K \cap R D)^{\circ}\right) N\left(D, s(K \cap S D)^{\circ}\right) & \leq N\left(D, \frac{s}{4}(K \cap R D)^{\circ}\right) .
\end{aligned}
$$

We successively apply Lemma 7 to the long products in (7) and (8). However, since for two neighboring factors in the products the hypothesis of Lemma 7 does not hold, an additional trick is required. We split the product into two parts, by grouping separately the factors corresponding to the odd and the even $j$ 's. If the growth of $\left(R_{j}\right)$ (which depends on the choice of $C_{2}$ ) is fast enough, the conditions of Lemma 7 are satisfied for each two consecutive odd factors, and for each two consecutive even factors. We thus arrive at

$$
N\left(D, C_{1} K^{\circ}\right) \leq N(K, D)^{C_{5}} \quad \text { and } \quad N\left(K, C_{1} D\right) \leq N\left(D, K^{\circ}\right)^{C_{5}},
$$

where $C_{5}=8 C_{4} C_{2}^{2}$ and $C_{1}$ are universal constants, which clearly yields (1). As indicated earlier, a (much) more careful analysis leads to a better constant $2+\varepsilon$ in the exponent, with $C_{1}=C_{1}(\varepsilon)$.

In fact, the last two steps of the proof give rise to the following proposition, valid for general symmetric convex bodies. It identifies the point in our scheme that is needed for generalizations beyond the euclidean case. (Our decision to include this statement in the form below was influenced by discussions with Nicole Tomczak-Jaegermann.)

Proposition 8 Let $T$ be a convex symmetric body in a euclidean space such that, for some constants $c, C>0$, the following holds: if $K$ is a convex symmetric body with $K \subset 4 T$, then

$$
N(K, T) \leq N\left(T^{\circ}, c K^{\circ}\right)^{C}
$$


Then, for some other constants $c^{\prime}, C^{\prime}>0$ (depending only on $c, C$ ) and any convex symmetric body $K$

$$
N(K, T) \leq N\left(T^{\circ}, c^{\prime} K^{\circ}\right)^{C^{\prime}} .
$$

Dually, if $K$ is fixed and the hypothesis holds for all T's verifying $K \subset 4 T$, then the assertion holds for any $T$.

Acknowledgment. This research was partially supported by grants from the US-Israel BSF (all authors) and the NSF [U.S.A.] (the third named author).

\section{References}

[A] S. Artstein, Proportional concentration phenomena on the sphere. Israel J. Math. 132 (2002) 337-358.

[AMS] S. Artstein, V.D. Milman, S.J. Szarek, More on the duality conjecture for entropy numbers. C. R. Math. Acad. Sci. Paris 336 (2003), no. 6, 479-482.

[KM] H. König, V. Milman, On the covering numbers of convex bodies, Geometric aspects of functional analysis (1985-86), Lecture Notes in Math., vol. 1267, Springer, Berlin-New York (1987) 82-95.

[L] M. Ledoux, Isoperimetry and Gaussian analysis. Lectures on probability theory and statistics (Saint-Flour, 1994), 165-294, Lecture Notes in Math., 1648, Springer, Berlin, 1996.

[MS] V. D. Milman, S. J. Szarek, A geometric lemma and duality of entropy numbers, Geometric aspects of functional analysis (1996-2000), Lecture Notes in Math., vol. 1745, Springer, Berlin-New York (2000) 191-222.

[P] A. Pietsch, Theorie der Operatorenideale (Zusammenfassung), Friedrich-SchillerUniversität Jena, 1972.

[T] N. Tomczak-Jaegermann, Dualité des nombres d'entropie pour des opérateurs à valeurs dans un espace de Hilbert, C. R. Acad. Sci. Paris Sér. I Math. 305 (1987), no. 7, 299-301. 